\title{
ARTEMiS (Automated Robotic Terrestrial Exoplanet Microlensing Search) - Hunting for planets of Earth mass and below
}

\author{
Martin Dominik $^{1} \dagger$, Keith Horne ${ }^{1}$, Alasdair Allan ${ }^{2}$, \\ Nicholas J. Rattenbury ${ }^{3}$, Yiannis Tsapras ${ }^{4}$, Colin Snodgrass ${ }^{5}$, \\ Michael F. Bode ${ }^{4}$, Martin J. Burgdorf ${ }^{4}$, Stephen N. Fraser ${ }^{4}$, \\ Eamonn Kerins ${ }^{3}$, Christopher J. Mottram ${ }^{4}$, Iain A. Steele ${ }^{4}$, \\ Rachel A. Street ${ }^{6}$, Peter J. Wheatley ${ }^{7}$ and Lukasz Wyrzykowski ${ }^{8,9}$ \\ ${ }^{1}$ SUPA, University of St Andrews, School of Physics \& Astronomy, \\ North Haugh, St Andrews, KY16 9SS, United Kingdom \\ email: (md35,kdh1)@st-andrews.ac.uk \\ ${ }^{2}$ School of Physics, University of Exeter, \\ Stocker Road, Exeter EX4 4QL, United Kingdom \\ email: aa@astro.ex.ac.uk \\ ${ }^{3}$ Jodrell Bank Centre for Astrophysics, The University of Manchester, \\ Manchester, M13 9PL, United Kingdom \\ email: (Nicholas.Rattenbury,Eamonn.Kerins)@manchester.ac.uk \\ ${ }^{4}$ Astrophysics Research Institute, Liverpool John Moores University, \\ Twelve Quays House, Egerton Wharf, Birkenhead, CH41 1LD, United Kingdom \\ email: (yt,mfb,mjb,snf,cjm,ias)@astro.livjm.ac.uk \\ ${ }^{5}$ European Southern Observatory (ESO), \\ Casilla 19001, Santiago de Chile, Chile \\ email: csnodgra@eso.org \\ ${ }^{6}$ Las Cumbres Observatory Global Telescopes Network, \\ 6740B Cortona Dr, Goleta, CA 93117, United States of America \\ email: rstreet@lcogt.net \\ ${ }^{7}$ Department of Physics, University of Warwick, \\ Coventry, CV4 7AL, United Kingdom \\ email: P.J.Wheatley@warwick.ac.uk \\ ${ }^{8}$ Institute of Astronomy, University of Cambridge, \\ Madingley Road, Cambridge CB3 0HA, United Kingdom \\ email: wyrzykow@ast.cam.ac.uk \\ ${ }^{9}$ Warsaw University Astronomical Observatory, \\ Al. Ujazdowskie 4, 00-478 Warszawa, Poland
}

\begin{abstract}
Gravitational microlensing observations will lead to a census of planets that orbit stars of different populations. From 2008, ARTEMiS will provide an expert system that allows to adopt a three-step strategy of survey, follow-up and anomaly monitoring of gravitational microlensing events that is capable of detecting planets of Earth mass and below. The SIGNALMEN anomaly detector, an integral part, has already demonstrated its performance during a pilot season. Embedded into eSTAR, ARTEMiS serves as an open platform that links with existing microlensing campaigns. Real-time visualization of ongoing events along with an interpretation moreover allows to communicate "Science live to your home" to the general public.
\end{abstract}

Keywords. gravitational lensing, planetary systems 


\section{Introduction}

While the overwhelming majority of all exoplanets known so far are gas giants, and most of these are in close orbits around their respective host star, the last few years have seen the detection of the first rocky or icy planets, with subsequently more Earthlike ones. The increasing sensitivity of several techniques will soon lead to exploring the abundance of planets that could harbour life. However, an understanding of their origin will only arise once models of planet formation and orbital migration can be made to match observations over an embracing wide region of parameter space, thereby providing a test of sufficient power to distinguish between alternatives.

The technique of gravitational microlensing is currently unique in its capability to provide a sample of terrestrial exoplanets at galactic distances rather than just in the Solar neighbourhood, and moreover can probe planets in wider orbits, whose orbital periods prevent them being detected by other indirect techniques. In particular, a planetary census for different stellar populations, namely the Galactic disk and bulge, can be obtained, and even the abundance of planets around stars in neighbouring galaxies, such as M31, can be determined (Covone et al. 2000; Chung et al. 2006).

With several planets having been detected, the most spectacular one of about 5 Earth masses (Beaulieu et al. 2006; Dominik et al. 2006), microlensing has become wellestablished. As shown here, with ground-based observations, it competes for the first detection of an Earth-mass planet against space missions such as COROT or Kepler, and it could even go further below in mass.

\section{Revealing exoplanets by microlensing}

Gravitational microlensing is understood as the transient brightening of an observed star due to the bending of light caused by the gravitational field of a foreground star. A substantial effect requires the source star at distance $D_{\mathrm{S}}$ and the lens star with mass $M$ at distance $D_{\mathrm{L}}$ to be located within an angle $\theta \lesssim \theta_{\mathrm{E}}$ on the sky, where

$$
\theta_{\mathrm{E}}=\sqrt{\frac{4 G M}{c^{2}}\left(D_{\mathrm{L}}^{-1}-D_{\mathrm{S}}^{-1}\right)}
$$

is the angular Einstein radius (Einstein 1936). For source stars in the Galactic bulge, gravitational microlensing events last about a month, while source and lens star carry out their transverse motion according to the kinematics of the Milky Way.

A planet orbiting the lens star can reveal its existence by causing a short deviation to the observed light curve that is otherwise symmetric with respect to a peak, preferably if it is separated from its host star by an angle $\delta \sim \theta_{\mathrm{E}}$ (Mao \& Paczyński 1991). Depending on the mass of the planet, the relative proper motion between the lens and source star, and the angular size of the source star, such planetary anomalies last between several hours and two weeks. Therefore, they constitute a snapshot of the planet, irrespective of its orbital period. Given that typically $\theta_{\mathrm{E}} \sim 350 \mu$ as and $D_{\mathrm{L}} \sim 6.5 \mathrm{kpc}$, microlensing is most sensitive to the detection of planets at an orbital separation of 1 to $10 \mathrm{AU}$.

With only about one in a million monitored stars being significantly brightened by the gravitational field of a foreground star at a given time (Kiraga \& Paczyński 1994), the OGLE (Optical Gravitational Lensing Experiment) and MOA (Microlensing Observations in Astrophysics) surveys monitor more than 100 million stars on a daily basis, which results in 700-1000 microlensing events per year being alerted on-line while they are in progress (Udalski 2003; Bond et al. 2001). While their sampling is however insufficient for detecting planets with masses significantly below that of Jupiter (Snodgrass 
et al. 2004), PLANET (Probing Lensing Anomalies NETwork) established the first microlensing follow-up network that was both capable of hourly sampling and a round-theclock coverage on selected events (Dominik et al. 2002). While this network of $1 \mathrm{~m}$-class telescopes relies on human observers and dedicated observing time, the demand of not only an immediate response, but also a flexible scheduling makes robotic telescopes ideally suited to carry out such an observing programme. Since 2004 - and since 2005 in cooperation with PLANET -, microlensing observations have been carried out with the RoboNet-1.0 network of UK-built $2 \mathrm{~m}$ robotic telescopes (Burgdorf et al. 2007), the largest of their kind. In contrast to PLANET/RoboNet, the MicroFUN team concentrate on a few quite promising events, with a network only being activated on target-of-opportunity basis.

The success of discovering a planet by microlensing critically depends on the availability of a telescope in a suitable location at the right time, which can mean within 10 min. To encourage follow-up observations, microlensing campaigns are therefore releasing photometric data in real time. For ongoing planetary anomalies, world-wide efforts are being undertaken to make sure that sufficient data are obtained, since there is no second chance.

\section{An anomaly detector to hunt for (sub-) Earth-mass planets}

In 2005, microlensing observations led to the discovery of OGLE-2005-BLG-390Lb, the first cool rocky/icy exoplanet ever found (Beaulieu et al. 2006; Dominik et al. 2006), with a mass of about 5 times that of Earth the least massive and most Earth-like exoplanet known at the time of its discovery. The light curve of the respective microlensing event OGLE 2005-BLG-390 showed a 15\% deviation over about a day due to the planet orbiting its lens star.

As Fig. 1 shows, an Earth-mass planet in the same spot would still have caused a signal amplitude of $\sim 3 \%$ and a duration of the planetary anomaly of $\sim 12 \mathrm{~h}$. While the standard follow-up sampling of $2 \mathrm{~h}$ would have been insufficient for characterizing the observed deviation and thereby claiming the presence of a planet, this would have been possible with high-cadence (10-15 min) anomaly monitoring, triggered upon the first suspicion of a departure from the symmetric ordinary light curve. Since 2007, the SIGNALMEN anomaly detector (Dominik et al. 2007) scans incoming microlensing data in real time for ongoing anomalies, where further observations are requested successively until an anomaly can be confirmed or rejected with the required significance.

By means of robust-fitting techniques, SIGNALMEN eliminates the effect of outliers, and triggers on residuals whose absolute value is among the upper $5 \%$ of all residuals for the respective site. Thereby, the fact that reported photometric error bars frequently do not properly represent the true uncertainties and in general do not follow a Gaussian distribution is addressed.

The source star in event OGLE-2005-BLG-390 was identified as a clump giant of spectral type G4 III with a radius $R_{\star} \sim 9.6 R_{\odot}$. As compared to a main-sequence star, this led to a larger probability to detect a planetary signal and increased its duration, but reduced its amplitude. Provided that the exposure times are chosen long enough for achieving a photometric accuracy of 1-2\%, SIGNALMEN also allows to reveal an Earthmass planet from a $5 \%$ deviation for a main-sequence source star $\left(R_{\star} \sim 1.2 R_{\odot}\right)$. While the microlensing searches face a fair chance of detecting an exoplanet of Earth mass within two years, the detection of planets with masses as small as $0.1 M_{\oplus}$ is challenging both by means of the short signal duration and the tiny probability for signals of appropriate amplitudes to occur, but nevertheless possible (Dominik et al. 2007). 

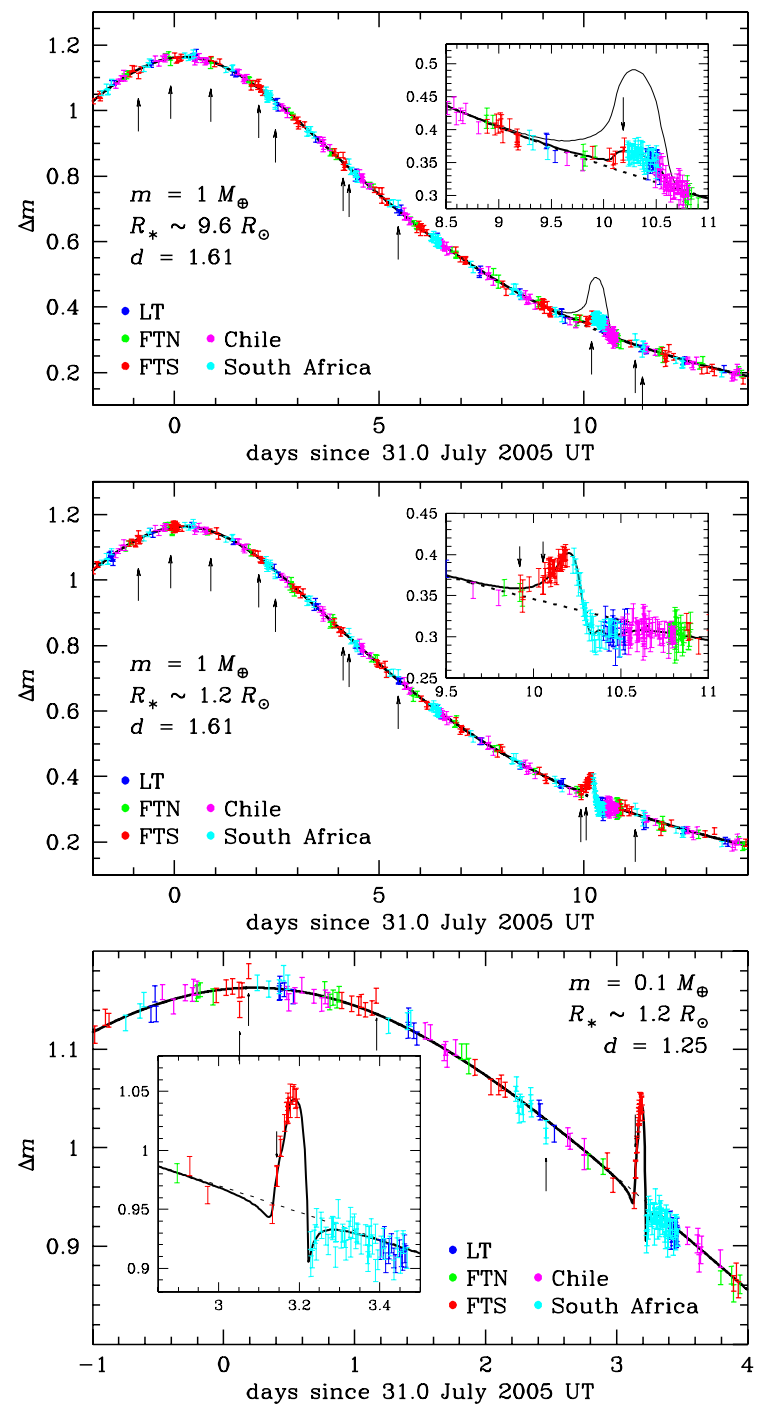

Figure 1. Simulations showing the possible detection of planets of Earth mass or below with the three robotic $2 \mathrm{~m}$-telescopes that constitute the RoboNet-1.0 network, namely the Liverpool Telescope (LT), the Faulkes Telescope North (FTN), and the Faulkes Telescope South (FTS), supplemented by two hypothetical further similar telescopes located in Chile and South Africa. For three different configurations, arrows indicate the epochs at which the SIGNALMEN anomaly detector requested further observations. (top) $1-M_{\oplus}$ planet in the same spot as OGLE-2005-BLG-390Lb $\left(d=\delta / \theta_{\mathrm{E}}=1.61\right)$, with the original model light curve also plotted; (middle) $1-M_{\oplus}$ planet in the same spot, but with a main-sequence source $\operatorname{star}\left(R_{\star} \sim 1.2 R_{\odot}\right.$ instead of $R_{\star} \sim 9.6 R_{\odot}$ ); (bottom) $0.1-M_{\oplus}$ planet at a closer distance $(d=1.25)$ to the lens star, and a main-sequence source star. For the middle and bottom panels, the orientation angle of the source trajectory has been adjusted for creating significant deviations. Light curves that would be observed in absence of any planets are shown as dashed lines.

\section{SIGNALMEN performance during the 2007 pilot season}

From April to October 2007, SIGNALMEN performed its pilot season, marking "phase zero" of ARTEMiS (Automated Robotic Terrestrial Exoplanets Microlensing Search), on observed data as these were released. SIGNALMEN provided 34 anomaly 
alerts on OGLE events that are clearly anomalous, and a further 3 on cases that are unclear due to the lack of follow-up data. Most notably, several candidate planetary signals have received crucial coverage as a result of SIGNALMEN flagging the respective event and the news being spread to the observing campaigns.

The art in designing a detection algorithm is in finding a suitable trade-off between the aims to maximize the number of correct detections, and to keep the number of false positives at a minimum. In particular, if very few false positives arise, the algorithm is almost certainly not well-balanced, but likely to be rather inefficient. After the elimination of obvious failures that can be grouped into 4 types that are fixed now by slight adjustments or further filtering, there were 3 false positives. While two of these refer to suggested signals at magnification $A \sim 1$, a required revision of the adopted model of an ordinary light curve has been mistaken for an anomaly in the remaining third case, which is a known and not unwelcome side-effect, because a dense sampling is required in order to sufficiently constrain the model parameters for keeping the opportunity for detecting anomalies (Dominik et al. 2007). For comparison, PLANET publicly circulated 35 anomaly alerts on OGLE events from 2004 to 2006, of which 4 were false positives. While SIGNALMEN detected a much larger number of anomalies per season, it provided a comparable fraction of false positives of $10-15 \%$.

SIGNALMEN did not fail to alert on any ongoing anomaly apparent by visual inspection that it should clearly have alerted on, while it considered insufficient evidence for an anomaly in 2 marginal cases. Coincidentially, it alerted on one real ongoing anomaly based on the false assumption of a significant deviation due to a shortcoming on finding an appropriate model, which is now fixed.

However, anomalies are missed under certain circumstances that deprive SIGNALMEN of any chance to detect an ongoing anomaly. In particular, SIGNALMEN cannot properly judge about anomalies that are already in progress with the first release of data on the respective event. Moreover, SIGNALMEN is designed to detect anomalies in progress in order to trigger the collection of further data that allow a proper characterization. If data are released in blocks rather than point-by-point, the anomaly may already be found over at the time of assessment, so that no ongoing anomaly can be reported. Finally, opportunities are missed when SIGNALMEN decides that an anomaly may be in progress, but an insufficient amount of data are subsequently collected for providing evidence before the potential anomaly is over. Given the fact that the microlensing surveys monitor substantially more events than current follow-up campaigns, the former harbour a potential for detecting anomalies, including such caused by planets, that so far is not efficiently exploited.

\section{The ARTEMiS concept}

In order to succeed with the detection of planets of Earth mass and below, several crucial requirements need to be met. Planetary anomalies in progress need to be sampled frequently, i.e. every $10 \mathrm{~min}$ or less, which also means that a telescope needs to be available in a suitable location. Moreover, the small probability for an Earth-mass planet to produce a deviation in a given event implies the need for many events to be monitored regularly. A brute-force approach would be a network of wide-field telescopes (e.g. Gould et al. 2007), carrying out a survey with a 10-min sampling, which comes with a substantial price tag for fully-dedicated facilities, and will not be realized in near time. However, a cooperative effort involving resources that already exist or are about to be commissioned, using a well-coordinated three-step strategy of survey, follow-up, and anomaly monitoring, is a serious alternative. 


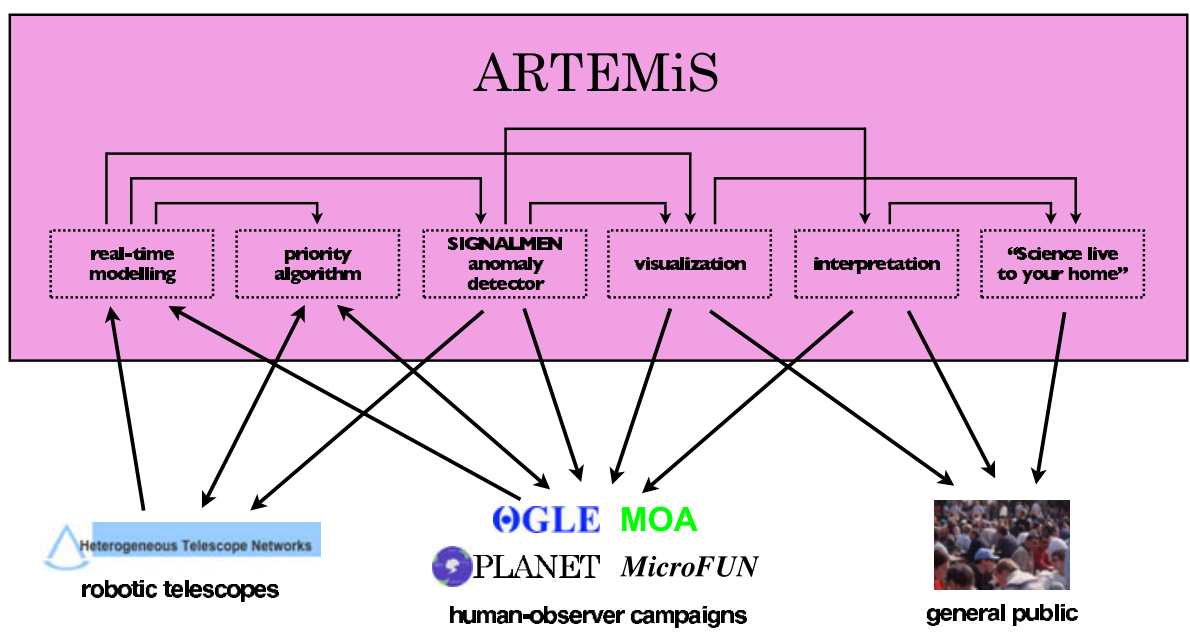

Figure 2. The components of ARTEMiS (Automated Robotic Terrestrial Exoplanet Microlensing Search) and how they interact with the outside world.

Even for an Earth-mass planet, a follow-up sampling interval of $1-1.5 \mathrm{~h}$ is sufficient for properly characterizing an anomaly from more frequent observations taken after a first trigger by SIGNALMEN. However, the current PLANET network of 1m-class telescopes is only able to monitor about $20 \%$ of all events found by the OGLE and MOA survey, and thereby falls short of exploiting their full potential for detecting planets. In contrast, a network of either $2 \mathrm{~m}$ or $4 \times 1 \mathrm{~m}$ telescopes at each site, of which RoboNet1.0 is a prototype, is well-suited to make full use of the capabilities of current surveys. Nevertheless, the participation of further smaller telescopes can critically decide about whether data can be obtained at crucial stages of an ongoing anomaly or not.

As shown in Fig. 2, ARTEMiS, whose first phase will go active in April 2008, bundles several components into an expert system that can coordinate the efficient detection of planets of Earth mass and below between microlensing observing campaigns using either robotic or staffed telescopes, and moreover provides outreach and live information to the general public.

In order to be able to react within a few minutes, ARTEMiS relies on real-time observational data being provided, which are then used to derive model parameters for the light curve of the respective event. Based on these models, the SIGNALMEN anomaly detector will decide on a suspected or ongoing anomaly and suggest further observations at specific sites, or circulate a public anomaly alert, respectively. The same real-time models are also used to prioritize the selection of ongoing events for follow-up monitoring by each observing site, which can define its specific capabilities and commitment, in order to maximize the expected number of planet detections. Moreover, ARTEMiS guides the human observers with the visualization of the collected data and model light curves as well as the interpretation of anomalous events. The selection of targets for follow-up and anomaly monitoring by means of a fully-deterministic automated procedure allows to predict the detection efficiency by carrying out simulations.

The ARTEMiS concept shares most of its paradigm with the eSTAR (e-Science Telescopes for Astronomical Research) project (Steele et al. 2002), which builds a metanetwork between existing proprietary robotic-telescope networks by providing a uniform interface based upon a multi-agent contract model (Allan et al. 2006). By embedding ARTEMiS into eSTAR and using the VOEvent (Virtual Observatory Event) protocol, 
a communication standard adopted by the IVOA (International Virtual Observatory Alliance), a direct interaction with the telescopes that are part of the HTN (Heterogeneous Telescope Networks) consortium becomes possible. Beyond that, ARTEMiS is also equipped with means of communication with human observers and the general public.

The real-time availability of both the data and their (preliminary) analysis allows to bring forefront science as a live event to the interested general public, who not only can watch a planet being discovered, but as amateur astronomers even can contribute to the discovery themselves.

\section{References}

Allan, A., Naylor, T., \& Saunders, E.S. 2006, Astronomische Nachrichten 327, 767

Beaulieu, J.-P., Bennett, D. P., Fouqué, P., Williams, A., Dominik, M., Jørgensen, U. G., Kubas, D., Cassan, A., Coutures, C., Greenhill, J., Hill, K., Menzies, J.,. Sackett, P. D., M. Albrow, Brillant, S., Caldwell, J. A. R., Calitz, J. J., Cook, K. H., Corrales, E., Desort, M., Dieters, S., Dominis, D., Donatowicz, J., Hoffman, M., Kane, S., Marquette, J.-B., Martin, R. Meintjes, P., Pollard, K., Sahu, K., Vinter, C., Wambsganss, J., Woller, K., Horne, K., Steele, I., Bramich, D. M., Burgdorf, M., Snodgrass, C., Bode, M., Udalski, A., Szymański, M. K., Kubiak, M., Wieckowski, T., Pietrzyński, G., Soszyński, I., Szewczyk, O., Wyrzykowski, Ł., Paczyński, B., Abe, F., Bond, I. A., Britton, T. R., Gilmore, A. C., Hearnshaw, J. B., Itow, Y., Kamiya, K., Kilmartin, P. M., Korpela, A. V., Masuda, K., Matsubara, Y., Motomura, M., Muraki, Y., Nakamura, S., Okada, C., Ohnishi, K., Rattenbury, N. J., Sako, T., Sato, S., Sasaki, M., Sekiguchi, T., Sullivan, D. J., Tristram, P. J., Yock, P. C. M., \& Yoshioka, T. 2006, Nature, 439, 437

Chung, S.-J., Kim, D., Darnley, M. J., Duke, J. P., Gould, A., Han, C., Jeon, Y.-B., Kerins, E., Newsam, A., \& Park, B.-G. 2006, ApJ, 650, 432

Bond, I. A., Abe, F., Dodd, R. J., Hearnshaw, J. B., Honda, M., Jugaku, J., Kilmartin, P. M., Marles, A., Masuda, K., Matsubara, Y., Muraki, Y., Nakamura, T., Nankivell, G., Noda, S., Noguchi, C., Ohnishi, K., Rattenbury, N. J., Reid, M., Saito, T., Sato, H., Sekiguchi, M., Skuljan, J., Sullivan, D. J., Sumi, T., Takeuti, M., Watase, Y., Wilkinson, S., Yamada, R., Yanagisawa, T., \& Yock, P. C. M. 2001, MNRAS, 327, 868

Burgdorf, M.J., Bramich, D.M., Dominik, M., Bode, M. F., Horne, K. D., Steele, I. A., Rattenbury, N., \& Tsapras, Y. 2007, Planetary \& Space Science, 55, 582

Covone, G., De Ritis, R., Dominik, M., \& Marino, A. A. 2000, A\&\&A, 357, 816

Dominik, M., Albrow, M. D., Beaulieu, J.-P., Caldwell, J. A. R., DePoy, D. L. , Gaudi, B. S., Gould, A., Greenhill, J., Hill, K., Kane, S., Martin, R., Menzies, J., Naber, R. M., Pel, J.-W., Pogge, R. W., Pollard, K. R., Sackett, P. D., Sahu, K. C., Vermaak, P., Watson, R., \& Williams, A. 2002, Planetary \& Space Science, 50, 299

Dominik, M., Horne, K., \& Bode, M. F. 2006, Astronomy \& Geophysics, 47, 3.25

Dominik, M., Rattenbury, N. J., Allan, A., Mao, S., Bramich, D. M.. Burgdorf, M. J.. Kerins, E., Tsapras, Y., \& Wyrzykowski, Ł. 2007, MNRAS, 380, 792

Einstein, A. 1936, Science, 84, 506

Gould, A., Gaudi, B. S., \& Bennett, D. P. 2007, Ground-based Microlensing Surveys, White paper submitted to the NASA/NSF Exoplanet Task Force, arXiv e-print 0704.0767

Kiraga, M., \& Paczyński, B. 1994, ApJ, 430, L 110

Mao, S., \& Paczyński, B. 1991, ApJ, 374, L37

Snodgrass, C., Tsapras, Y., \& Horne, K. 2004, MNRAS, 351, 967

Steele, I. A., Naylor, T., Allan, A., Etherton, J., \& Mottram, C. J. 2002, in: R. I. Kilbrick (ed.), Advanced Global Communications Technologies for Astronomy II, Proc. SPIE, Vol. 4845, p. 13

Udalski, A. 2003, AcA 53, 291 